FERMILAB-Conf-91/69

\title{
Shielding Calculations for SSC
}

\author{
A. Van Ginneken \\ Fermi National Accelerator Laboratory \\ P.O. Box 500 \\ Batavia, Illinois 60510
}

March 1990

*Presented at the Third Annual International Industrial Symposium on the Super Collider [ITSSC], Atlanta, Georgia, March 13-15, 1991. 


\title{
Shielding Calculations for SSC*
}

\author{
A. Van Ginneken \\ Fermi National Accelerator Laboratory ${ }^{\dagger}$ \\ P. O. Box 500, Batavia, IL 60510
}

March 1991

\begin{abstract}
Monte Carlo calculations of hadron and muon shielding for SSC are revieved with emphasis on their application to radiation safety and environmental protection. Models and algorithms for simulation of hadronic and electromagnetic showers, and for production and transport of muons in the $\mathrm{TeV}$ regime are briefly discussed. Capabilities and limitations of these calculations are described and illustrated with a few examples.
\end{abstract}

\section{Introduction}

Along with a few empirical rules of limited applicability almost all high energy accelerator shielding calculations rely on the Monte Carlo method. Combining all the information necessary to simulate particle production and transport from $\mathrm{TeV}$ down to $\mathrm{MeV}$ results in large code systems. From the shielding point of view, four basic types of particles (problems) are usually distinguished: (1) hadrons, mostly nucleons and pions, which in a proton accelerator are the primary group of particles, but treating separately: (2) low energy neutrons, produced in abundance when the higher energy particles interact with nuclei, (3) electrons and photons, derived mostly from the decay of $\pi^{0}$ which are produced in hadronic collisions, and (4) muons resulting mainly from $\pi^{ \pm}$or $D$ meson decay, but also produced in significant numbers by a host of other processes.

*talk ał IISSC Symposium, Atlanta, March 1991.

'Fermi National Accelerator Laboratory is operated by Universities Research Association under contract with the US Department of Energy. 
The main function of these codes is to trace the cascades, which typically develop when a high energy particle interacts in bulk matter, through some idealized version of the beam/target geometry and magnetic fields present in some apparatus or facility (real or fancied). The program we're most familiar with is CASIM [1] which was written at Fermilab and continues to see extensive use there. Others are, e.g., FLUKA [2] from CERN and MARS [3] written at Serpukhov. There exist also a number of codes to simulate detector response to high energy particle interactions, which is indispensible in the design and analysis of high energy experiments. Although they have a great deal in common, the needs of the two types of programs are quite different: shie]ding calculations can assume that a large number of (beam) particles is involved, correlations among outgoing particles can be ignored, a 'common sense' approach to questions of geometry and fields is generally sufficient. By contrast detector response codes consider individual events, fluctuations and correlations are extremely important and since a large detector operates in one-or at most a few-configurations one can take the time to represent the geometry, etc., in painstaking detail. Once patience will be rewarded by a smaller error in correcting for efficiencies, etc. For the above reasons there is only limited cross application of the two. Shielding type calculations might be useful in the preliminary stages of detector design but cannot simulate individual events. Detector codes can in principle be applied to shielding problems but are too slow and cumbersome for routine use. Their CPU time demands increase linearly with the accelerator energy vis-a-vis logarithmic for CASIM.

In recent years, shjelding calculations have seen extensive use in problems concerned with protection of essential or expensive equipment rather than personnel or the environment, e.g., problems of avoiding quenching of the superconducting magnets cite and of radiation damage to silicon detectors. Because most of the action takes place within the accelerator structure, these problems tend to pose a stiffer computational challenge vis-a-vis the more mundane health physics applications to which this review is addressed. Another important application of these codes which is omitted here is that of target heating, radiation induced shock waves, etc. This is important in the design of beam dumps and thereby has, at least tangentially, some environmental impact. Also omitted here are the paralegal aspects of this business - the laws and the recommendations on dose limits, what are the as. sumed routine and accidental beam loss conditions, et.c.,--which ultimately determine how much shielding must be provided. The remainder of this brief review is heavily biased towards CASIM, with apologies to the other codes 
and their authors as well as to the reader, for such lack of evenhandedness. It is also biased towards the high energy end, i.e., towards what is different at the SSC.

\section{Hadrons}

Hadron shielding deals with both the prompt ('beam on') radiation and with radioactivity produced in the environment around the accelerator. A key parameter for an accelerator or experimental area is the amount of lateral shielding required to reduce the hadron dose to an acceptable level. Especially around beam dumps there is the additional question of protecting the groundwater against induced radioactivity. It should be remembered that all such hadron shielding considerations boil down to shielding against low energy neutrons and an SSC calculation differs from one at Fermilab typically only with what happens in the first generation.

In any realistic shjelding situation one always averages over a large number of particles and one therefore gains enormously using weighted calculations for shielding (in contrast with, most notably, detector simulation). This results in significant economies in modeling--both conceptually and in the amount of coding. The chief advantages of the weighting are (1) hadron production is represented by inclusive distributions, (2) it allows one to concentrate on the particles which contribute most to the problem, and (3) by exponentially reducing the weight of a particle as it traverses the geometry it is well suited to study problems of deep penetration which are the essence of shielding problems. More detail on wejghted vs analog calculations may be found in refs. $[4$.

The particle production model used in the original version of CASIM [1 ? is the Hagedorn-Ranft model to which is added a high $p_{T}$ component and a low energy nucleon component. [5]. In terms of physics, the model is quite outdated but has a large number of loose parameters which have been tied to experiment so that it generally works well in the sub- $\mathrm{TeV}$ region. However it does not predict the multiplicities and other gross features observed, e.g., in $\bar{p} p$ collisions at CERN and Fermilab, very well. To semedy this the hadroproduction model in CASIM has recently been updated $[6]$ to reflect better what is now known from the hadron colliders and from theory. However evaluation of practical hadron shielding requirements is not very sensitive to what is basically only the first generation of the cascade chain [7] when care is taken to conserve energy and momentum and provided none of the 
other important gross features (multiplicity, limited $p_{T}, \pi^{0}$ inelasticity, etc.) are flagrantly misrepresented. Some general calculations 8 performed with the old model during the early SSC design stages are still expected to be a useful guide. At the low energy end, hadrons are followed only down to some cut-off ( $50 \mathrm{MeV}$ typically) which allows for considerable simplification of the code.

Hadron transport in the $\mathrm{TeV}$ regime differs from the lower energies in that direct electron pair production and bremsstrahlung become increasingly important mechanisms of energy loss. [9] This again affects only the first few generations of the cascade and is only of limited importance in hadron shielding calculations. While both processes have very broad energy loss distributions, they are adequately represented by an average $\mathrm{dE} / \mathrm{dx}$ in these calculations. For $\mathrm{TeV}$ pions bremsstrahlung becomes an important source of angular diffusion.

In a typical hadron shielding calculation one keeps track of the nuclear interactions $(\geq 50 \mathrm{MeV}$ ), occurring as a result of the cascade development, as a function of location throughout the target. Charged particles below this cut-off quickly slow down (though $\pi^{-}$in heavier targets will still interact in a nucleus). Neutrons below $50 \mathrm{MeV}$ can migrate considerably, though generally with a shorter interaction length and with limited multiplicative power. In heavy targets the largest source of neutrons is the 'evaporation' process of excited nuclei which have a typical energy of a few MeV. Particularly when hydrogen is present in the medium they lose energy fairly quickly and get absorbed. One envisions then a situation in which an equilibrium is established in the outer regions of the shield (generally soil or concrete) so that the dose may be predicted from the nuclear interaction (or 'star') density of the energetic $(\geq 50 \mathrm{MeV})$. A conversion factor $[10]$ is applied which is based on a low energy neutron spectrum calculated deep inside a soil shield. [11 Such spectra are rather insensitive of location and of their detailed origin. For limited radial shielding there may be considerable contribution from high energy hadrons and from electromagnetic showers, so that a simple conversion factor does not apply.

A typical hadron shielding calculation might start from ref. [8? plus some rules of thumb for a rough estimate of the requirements. One then proceeds to model (an over-shielded version of) the particular beam/target geometry of the facility in question. This last part varies from one calculation to the next and must be supplied by the user. In principle the only limit to how realistic a model to furnish, is one's patience. But in practice the point of diminishing returns is usually reached much earlier and the real skill is 
to distinguish what might be important from all the rest and to simplify as much as possible what remains. This is even more important in the design stages when what's being modeled is not yet firm. The star density is computed throughout the entire geometry. These are readily converted to dose and one can then decide on the correct shield dimensions.

The Monte Carlo is done in three dimensions but, where possible, the analysis (binning of stars in this example) is done assuming cylindrical symmetry. This is mostly for reasons of statistical accuracy since there can be fewer, larger bins (and one can leave the binning procedures of CASIM alone). Where this cannot be reasonably maintained one retreats (gingerly) to some lesser symmetry, perhaps four-fold: left/right and up/down symmetric, or cylindrical but with as few azimuthal bins as possible. Another reason for keeping down the number of bins is to make the information generated more presentable (and digestible). For a simple geometry one can resort to contour plots such as shown below but this may not be suitable for more complicated geometries. Fig. 1 shows an example of such a calculation from the collection of ref. [8]. The star density is also easily converted into a dose from residual radioactivity. A CASIM derived dose can serve as input for calculations of transmission through labyrinths, etc. Ref. '12' cites some of the comparisons of CASIM with observation at Fermilab. On the whole such comparisons have been gratifying.

\section{Electrons and Photons}

As mentioned above, $\gamma$ and $e^{ \pm}$originate mainly from the decay of $\pi^{0}$ produced in hadron cascades. Although other sources have been identified they are negligible by comparison. The $\pi^{0}$ decays into $2 \gamma$ which converts into $\epsilon^{ \pm}$pairs when traveling through bulk matter and the $e^{-}$or $e^{+}$in turn emit $\gamma \mathrm{s}$ by bremsstrahlung. This multiplicative behavior leads to an electromagnetic shower. In the typical hadron shield such a shower get absorbed long before the neutrons are, and they can therefore be neglected in most dose calculations. This is not the case for radiation problems with accelerator components or beam dumps and for this purpose AEGIS [13], a companion to CASIM, has been written also using the weighted Monte Carlo approach. The physics of the underlying processes has been known for at least fifty years and although some new effects are predicted to occus at multi- $\mathrm{TeV}$ energies these will not yet have any practical significance at the SSC. Pro-

duction of hadrons by the electromagnetic shower, while of some consequence 


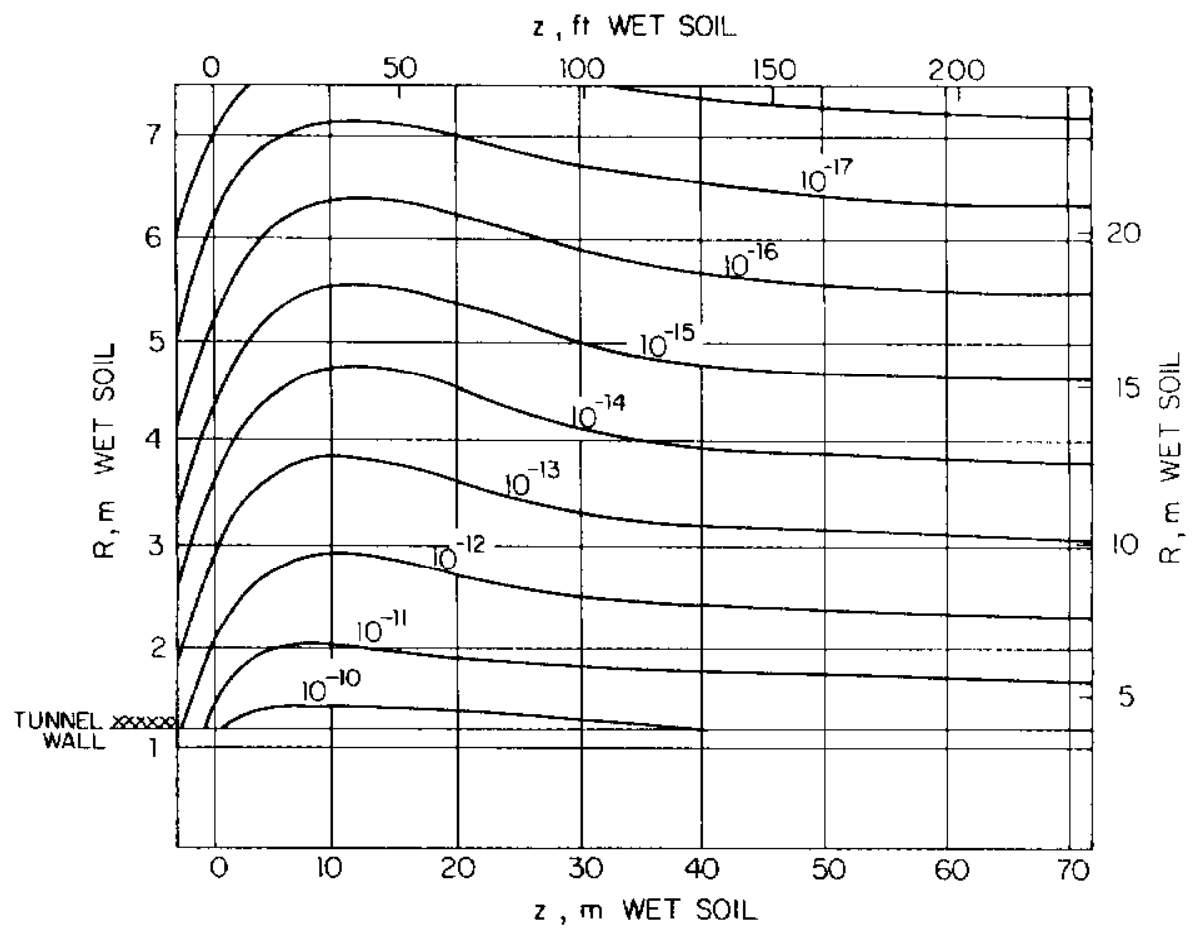

Figure 1: Iso-dose contours (rem/interacting proton) in soil around tunnel when $20 \mathrm{TeV}$ protons are lost on the side of a bare beampipe.

around electron accelerators, is negligible compared to that by the hadrons themselves. Muon production by these showers has some significance. There are a varjety of mechanisms (see below), but the most copious one is photon conversion into $\mu^{ \pm}$.

\section{Muons}

As far as hadrons, electrons, and photons are concerned shielding at the SSC is just a bit 'more of the same'. The extra shielding required is measured in feet. Muon shielding however introduces a real qualitative difference in scale. If, for example, we take muons of one tenth of the incident energy as being produced in significant numbers: it takes about $200 \mathrm{~m}$ of soil to stop a muon of $0.1 \mathrm{GeV}$ (at Fermilab) but about $1600 \mathrm{~m}$ to stop a $2 \mathrm{TeV}$ muon (SSC). Moreover the $2 \mathrm{TeV}$ muon shows much more straggling and one still expects to see some at $2500 \mathrm{~m}$. For these reasons site criteria, land acquisition, etc. for the SSC are largely a direct result of muon shielding consideration. In 
contrast to hadron shielding calculations one cannot resort to armwaving about conservation laws, etc., to make things easier. The muons one must shield against come typically from the first few generations of the combined hadron and electromagnetic cascade and on average they carry off only a tiny fraction of the energy of the beam particles. Muon transport is for the most part based on 'old' physics but a shift in emphasis among the different processes responsible for muon energy loss and angular diffusion takes place as the muon reaches the $\mathrm{TeV}$ regime. $[9]$ As a result fiuctuations become much more important, leading to the aforementioned increase in straggling.

Muon production is usually split into (1) muons from $\pi$ and $K$ decay and (2) 'prompt' muons (which includes decays of short lived particles). This division has practical consequences: the $\pi$ and $K$ decay times are long enough to make this component very sensitive to the geometry and composition of the shield. In bulk matter its magnitude will increase (almost linearly) with the nuclear int eraction length. Moreover, around an accelerator one typically finds large regions of negligible density (beam aperture, tunnels, etc.) where $\pi \mathrm{s}$ and $K \mathrm{~s}$ have the opportunity to decay without removal by nuclear interactions. As their name suggests prompt muons are, at least for our purpose, produced instantaneously and therefore do not exhibit this extra sensitivity to geometry and composition. Hadronically produced prompt muons which are presently included in CASIM calculations derive from (a) D meson decay, (b) vector mesons $(\rho, \omega, \phi, J / \psi)$ decaying into $\mu^{+} \mu^{-}$, and (c) dimuon continuum both from Drell-Yan and 'soft annihilation' mechanisms. Electrons and photons are assumed to produce muons via (d) pair creation by photons, (e) decay of vector mesons produced by photons on nuclear targets (coherent, incoherent, and inelastic production), and (f) annihilation of $e^{-}$ with atomic electrons. There are still large uncertainties involved in prompt muon production particularly from soft annihilation in the TeV regime. The representations in CASIM are based mostly on experiment and the models are used just as convenient tools of extrapolation.

The electromagnetic processes have typically much lower cross sections than the hadronic ones yet they are important since (1) with increasing primary energy ( $20 \mathrm{TeV}$ at the SSC) an increasing fraction of this energy is spent on producing electromagnetic showers at the expense of the hadronic part, and (2) in mechanisms (d-f) essentially all the energy of the $\gamma$ or $e^{+}$ converts into energy for the $\mu^{+} \mu^{-}$pair (the inelastic part of (e) is the sole exception). The hadronic processes are much less energy efficient in this regard.

At 'low' energies ( $E_{\mu} \leq 300 \mathrm{GeV}$, say) the muons lose energy predomi- 
nantly via collision with atomic electrons and change their direction mostly by multiple Coulomb scattering. Slowing down and angular diffusion can be to good approximation handled on a continuous basis. Above a few TeV, on the other hand, one finds that energy loss by direct $\left(e^{\dagger} e^{-}\right.$pair production, bremmstrahlung, and deep inelastic scattering (in that order) all exceed atomic collisions as a source of energy loss. As for angular spreading, bremsstrahlung becomes the most important process while deep inelastic scattering becomes competitive with multiple Coulomb scattering and pair production is still negligible. The above statements refer to averages only but to get the straggling right most of muon transport must be simulated on an event-by-event basis.

Muons from the initial collision, e.g., of a $20 \mathrm{TeV}$ proton with an iron nucleus or of 20 on $20 \mathrm{TeV}$ protons, usually form a significant part of a muon shielding problem. But to get the full picture one must include muons from all generations of the hadronic and electromagnetic cascade. In CASIM both prompt muons are created in each generation while decay muons are produced in weighted fashion, depending on the length of the meson trajectory. Because muons below a few $\mathrm{GeV}$ are absorbed by a typical hadron shield one can run with a higher energy cut-off. The muons are then transported using a continuous approximation only for atomic collisions and for multiple Coulomb scattering. The other processes listed above, plus energetic collisions with electrons ( $\delta$ rays), are considered as individual events. For each such process a variable energy loss is first randomly selected from the appropriate distribution. For a given a particular energy loss an rms angular deflection is determined in the manner of ref. [9] whence a particular angular deflection is randomly chosen from a Gaussian.

The strong dependence of the $\pi / K$ decay component on the geometry makes life a little more difficult in that one has to rely even more (relative to hadron shielding) on Monte Carlo and less on rules of thumb. This is all the more true when magnetic fields are present since, e.g., muons can follow the beam aperture for long distances being alternatively swept from the beampipe onto the return field of the magnet and thence bumped back into the aperture. While a CASIM calculation will include such effects, these facts make generalizations difficult including questions of what is a "worst case' beam loss accident and what is its associated dose at a given location. Fig. 2 shows an example of a muon dose calculation for the contemplated Large Hadron Collider at CERN. 


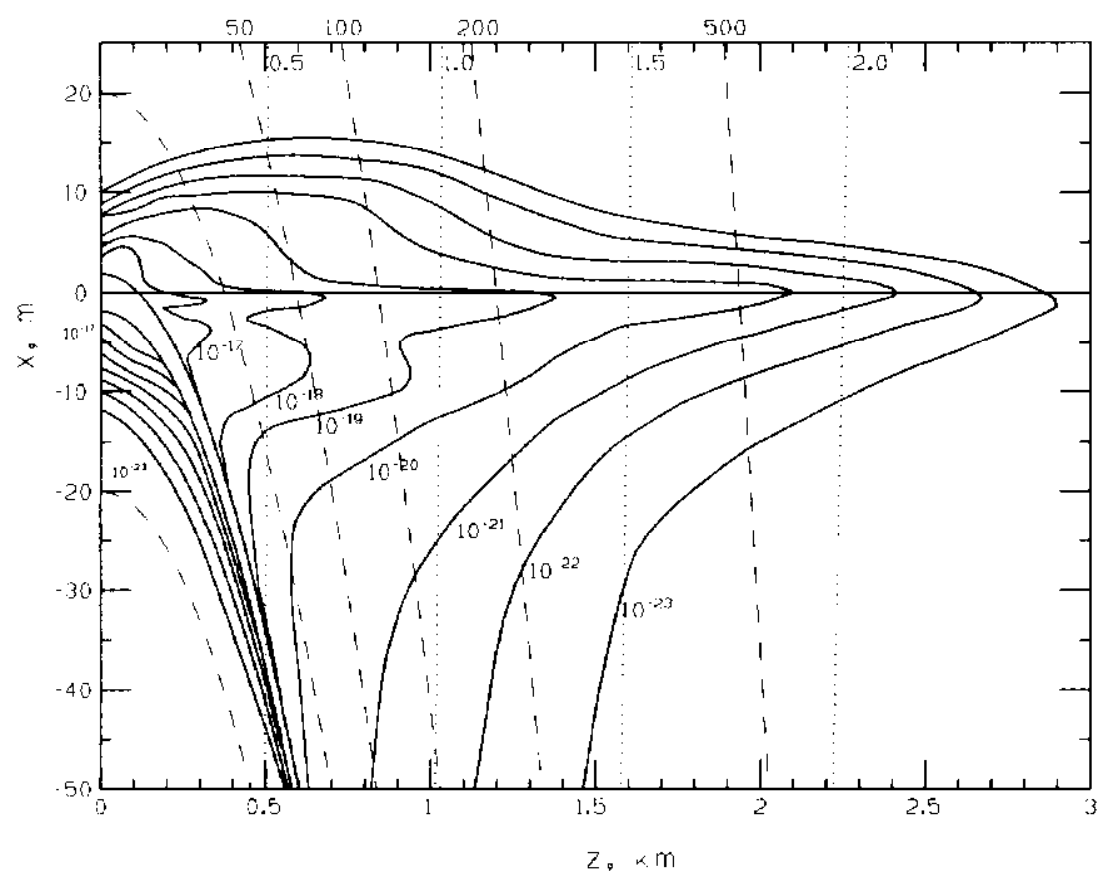

Figure 2: Iso-dose contours (sievert/interacting proton) in median plane in soi] around tunnel when $8 \mathrm{TeV}$ protons are lost on the out-side of the beampipe in a continuous dipole.

\section{Special CASIM Calculations}

The foregoing summary describes what is a vailable to the more or less casual user. By programing for a particular geometry the results of such a calculation allow the dose to be determined as a function of location throughout the entire geometry. The biasing techniques, etc., applied in CASIM aim to do a good job for such general purpose calculations. While there are a number of options available in the user supplied data (energy cut-off, collision length biasing, etc.) one may occasionally make changes in the program itself to efficiently perform more specjalized demands. A user may wish to know, e.g., the energy spectrum of positive muons at one or more specific locations. An example from real life is that of antiproton production and transport, to help with the design of the Fermilab Antiproton Source, [14] which required some extra routines to deal with the $\bar{p} \mathrm{~s}$. A recent addition to CASIM is that of smoothing the final results. [15] This is useful in the many 


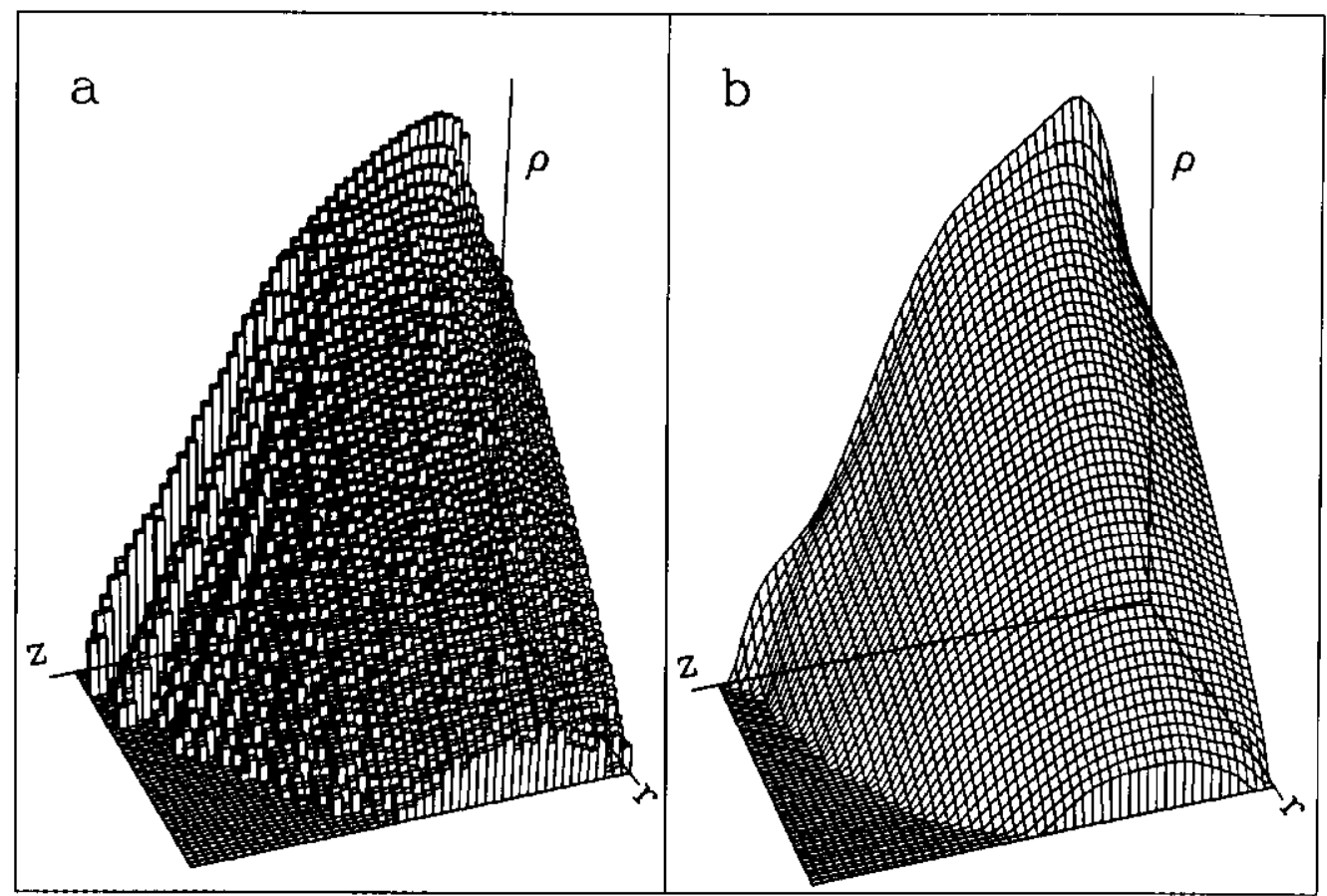

Figure 3: (a) Two dimensional histogram of CASIM dose calculation, and (b) smooth approximation. Vertical scale is logarithmic and extends over about twenty orders of magnitude.

cases where there are still statistical difficulties in regions where one must know the dose. Presently one gets around this by averaging over a larger region but it is more convenient and probably less biased to automate such a procedure. Fig. 3 shows an example of such smoothing. There has not been much experience with it yet.

\section{Concluding Remarks}

Shielding calculations, whether by CASIM or other such programs $[2,3]$, appear in good shape to meet the SSC's demands of radiation and environmental protection. There are some uncertainties, e.g., in muon production, but we know from cosmic ray studies that there will be no big surprises. For fixed target SSC we know this in even more detail from CERN and Fermilab collider results. But it will still be very ineteresting to compare predictions with results at SSC, particlularly for simple geometries, such 
as a dump. Since the demands for radiation safety appear to be growing with time (lower dose limits, higer quality factors, etc.) there are increased demands on the speed with which a particular geometry can be analyzed: faster computers, faster algorithms, and faster ways to interpret the results.

\section{References}

[1] A. Van Ginneken, Fermilab-FN-272 (1975).

[2] P. Arnio et al., CERN TIS-RP/168 (1986).

[3] N. V. Mokhov, IHEP preprint 82-168, Serpukhov (1982).

44. A. Van Ginneken, Calculation of the Average Properties of Electromagnetic Cascades at High Energies (AEGIS), p. 211 in Computer Techniques in Radiation Transport and Dosimetry, $\mathrm{W}$. R. Nelson and T. M. Jenkins, Eds.,Plenum Publishing Corp., New York (1980) and Calculation of the Average Properties of Hadronjc Cascades at High Energies (CASIM), ibid., p. 323.

5: J. Ranft and J. T. Routti, CERN-II-RA/72-8 (1972).

6] S. Qian and A. Van Ginneken, Fermilab FN-514 (1989).

[7] For problems involving colliding beams the production model used for pp collisions is a simple empirical model based on extending observations from CERN and Fermilab colliders and on calculated results [J. Ranft, DTUJET, p. 67 in Radiation Levels in SSC Interaction Regions, D. E. Groom, Ed., SSC Central Design Group (1988). For more detail see ref. [6].

[8] A. Van Ginneken et al., Fermilab FN-447 (1987).

9. A. Van Ginneken, Nucl. Inst. Meth., A251, 21 (1986).

$[10$ A. Van Ginneken and M. Awschalom, High Energy Particle Interactions in Large Targets, Fermilab, Batavia, IL (1975).

11: T. A. Gabriel and R. T. Santoro, ORNL-TM-3262 (1970).

12] M. Awschalom et al., Nucl. Inst. Meth., 131, 235 (1975); M. A wschalom et al., ibid., 138, 521 (1976); J. D. Cossairt et al., ibid., 197, 465 
(1982); J. D. Cossairt et al., ibid., A238, 504 (1985); N. V. Mokhov and J. D. Cossairt, ibid., $A 244,349$ (1986).

[13, A. Van Ginneken, Fermilan FN-272 (1975).

14] C. Hojvat and A. Van Ginneken, Nucl. Inst. Meth., 206, 67 (1983).

[15] A. Van Ginneken, Smoothing Algorithm for Histograms of One or More Dimensions, Fermilab Pub-90/197 (1990), to be published in Nucl. Inst. Meth. 